\title{
Improved Group Acceptance Sampling Plan for Dagum Distribution under Percentiles Lifetime
}

\author{
Muhammad Aslam ${ }^{1, a}$, Muhammad Shoaib ${ }^{b}$, Hina Khan ${ }^{b}$ \\ ${ }^{a}$ Department of Statistics, Forman Christian College University \\ ${ }^{b}$ Department of Statistics, GC University
}

\begin{abstract}
This paper deals with a group acceptance sampling plan for time truncated tests which are based on the total number of failures from the whole group assuming that the life time of an item follows the Dagum (inverse Burr) distribution. This study is developed when a multiple number of items as a group can be tested simultaneously in a tester. The minimum number of groups required for a given group size and acceptance number is determined such that the producer and consumer risks are satisfied simultaneously at the specified quality level, while the termination time and the number of testers are specified. Comparisons are made between the proposed plan and the existing plan on the basis of size of the groups. Two real examples are provided.
\end{abstract}

Keywords: Group acceptance sampling plan, Dagum distribution, consumer risk, producer risk, truncated life test.

\section{Introduction}

Various techniques of inspection exist in SQC to ensure or improve the quality of a product to required standards. To meet the rapid advancement, the manufacturer wants to use the high quality manufacturing technology. Acceptance sampling is one of the most important techniques of statistical quality control to ensure the quality of the product. Acceptance sampling is an important tool to judge the quality of the product inspected in a sample taken from the lot and on the basis of this judgment make a decision to accept or reject a submitted lot of the product.

Accepting sampling is often used when the inspection of the product is too costly. Acceptance sampling is reducing the complete inspection also minimizes cost and the time. It is implicitly assumed in the usual sampling plans that only a single item is put in a tester. Acceptance sampling on the basis of single item by using the various lifetime distribution are discussed by many authors Epstein (1954), Goode and Kao (1961), Kantam and Rosaiah (1998), Kantam et al. (2001), Baklizi (2003), Rosaiah et al. (2006), Rosaiah and Kantam (2005), Tsai and Wu (2006), Rosaiah et al. (2007), Aslam and Shahbaz (2007), Aslam and Kantam (2008), Aslam (2008) and Balakrishnan et al. (2007), Aslam and Jun (2010). However, the tester wants to test multiple numbers of items at a time because testing cost and time can be saved by testing these items simultaneously. This type of testers is frequently occurred in the sudden death testing by Pascual and Meeker (1998) and Vlcek et al. (2003), Jun et al. (2006). The sampling plan under this type of tester will be called a group acceptance sampling plan under the truncated life test. According to Aslam and Jun (2009b) a sampling plan with this type of tester determines that the sample size is equivalent to determining the number of testers. If we call items in a testers as a group and then determine the required number of groups.

\footnotetext{
${ }^{1}$ Corresponding author: Professor, Department of Statistics, Forman Christian College University, Lahore 5400, Pakistan.

E-mail: aslam_ravian@hotmail.com
} 
Aslam and Jun (2009b) developed the group acceptance sampling plan on the truncated life test when the lifetime of a product follow Inverse Rayleigh or log-logistic distribution and Srinivasa Rao (2009) developed the Marshall-Olkin Extended Lomax distribution using a single point approach.

In the literature, a group acceptance sampling plan or ordinary acceptance sampling plan is developed by using the mean or median lifetime; however, in this paper uses the percentile lifetime to assure the quality of the product. Lio et al. (2010a, 2010b) develop the acceptance sampling plan by using percentile lifetime to meet consumer expectations. Percentile lifetime is best fitted on the skewed distribution as compared to mean lifetime.

The role of consumer and producer risks in a group acceptance sampling plan is very important to meet the required standards of the product. The probability of accepting the defective lot is called the consumer risk and the probability of rejecting the good lot is called the producer risk. Aslam and Jun (2009b) develop the group acceptance sampling plan by satisfying only the consumer,s risk. In this paper we use the two point approach to satisfy consumer and producer risks. The two point approach on the OC-curve for designing the variable acceptance sampling plan has been developed by Fertig and Mann (1980) and Jun et al. (2006).

The main purpose of this paper is to design the group acceptance sampling plan for the truncated life test when the lifetime of an item follows a Dagum distribution under percentiles lifetime with a known or unknown shape parameter. We obtain the required number of groups and the acceptance number simultaneously for the given values of consumer's and producer's risks.

\section{Introduction to Distribution}

The Dagum distribution is introduced by Dagum (1977) and is also known as the Inverse Burr distribution. The Dagum distribution has positive asymmetry. The Dagum distribution is a lifetime distribution and is also used in the field of reliability to check the survival time of the data by Domma et al. (2009). Suppose that the lifetime of an item or a product follows a Dagum distribution, where the probability density function(pdf) and cumulative distribution function(cdf) is given by:

$$
\begin{aligned}
& f(t)=b \lambda \delta t^{-\delta-1}\left(1+\lambda t^{-\delta}\right)^{-b-1}, \quad t>0, b>0, \lambda>0, \delta>0, \\
& F(t)=\left(1+\lambda t^{-\delta}\right)^{-b}, \quad t>0, b>0, \lambda>0, \delta>0,
\end{aligned}
$$

where $\lambda$ is the scale parameter, $b$ and $\delta$ are the shape parameters. The $100 q^{\text {th }}$ percentile of the Dagum distribution from Equation (2.2) is given by:

$$
t_{q}=\lambda^{\frac{1}{\delta}}\left(q^{-\frac{1}{b}}-1\right)^{-\frac{1}{\delta}}
$$

Particularly, the $50^{\text {th }}$ percentile from equation (2) is given by:

$$
m=\left[\frac{1}{\lambda}\left\{(0.5)^{-\frac{1}{b}}\right\}-1\right]^{-\frac{1}{\delta}} .
$$

\section{Proposed Group Sampling Plan Based on Total Number of Failures}

In the literature, Aslam and Jun (2009a, 2009b) proposed the group acceptance sampling plan and made a decision on the basis that a lot under inspection will be accepted if the number of defective items in each group is smaller than or equal to the specified number, otherwise the lot is rejected. By using this source, we propose the following group sampling plan based on the total number of failures. 
Let $t_{q}$ represent the true percentile life of a product and $t_{q_{0}}$ denote the specified percentile life. A product is stated as good and accepted for consumer use if the sample information satisfies the hypothesis $H_{0}=t_{q} \geq t_{q_{0}}$ : otherwise, the lot of the product is rejected. In acceptance sampling procedure, this hypothesis is tested based on the number of defectives (failures) from a sample in a prefixed time. The lot is rejected if the number of failures exceeds the acceptance number $c$. The hypothesis $t_{q} \geq t_{q_{0}}$ is providing enough evidence at certain level of both risks to accept the lot. The proposed group acceptance sampling plan under the total number of failures is stated below:

1. Draw the random sample of size $n$ from a lot, allocate $r$ items to each of $g$ groups (or testers) so that $n=r g$ and put them on test for the duration of $t_{0}$.

2. Accept the lot if the total number of failures from $g$ groups is smaller than or equal to $c$. Truncate the test and reject the lot as soon as the total number of failures from $g$ groups is larger than $c$ before $t_{0}$.

It is important to note that the proposed group plan is the generalization of the ordinary single acceptance sampling plan. If $r=1$, this plan becomes the ordinary single sampling plan.

If the total number of failures occurred from all groups is smaller than or equal to the specified action limit $c$. Therefore, the lot acceptance probability of the proposed plan is given by:

$$
L(p)=\sum_{i=0}^{c}\left(\begin{array}{c}
r g \\
i
\end{array}\right) p^{i}(1-p)^{r g-i},
$$

where $p$ is the probability that an item in any group fails before the termination time $t_{0}$. It would be convenient to write the termination time $t_{0}$ as a multiple of the specified life $t_{q_{0}}$ and termination ratio $\delta_{q}$. That is, we will consider $t_{0}=\delta_{q} t_{q_{0}}$ for a constant $\delta_{q}$.

The Dagum distribution under the $100 q^{\text {th }}$ percentile is given by:

$$
p=\left[1+\left\{\frac{1}{\gamma \delta_{q}}\left(\frac{t_{q}}{t_{q_{0}}}\right)\right\}^{\delta}\right]^{-b},
$$

where $\gamma=\left(q^{-1 / b}-1\right)^{-1 / \delta}$ and $t_{q} / t_{q_{0}}$ is the ratio of true percentiles life to the specified percentiles life.

The Dagum distribution under the $50^{\text {th }}$ percentile is given by:

$$
p=\left[1+\left\{\frac{1}{a \gamma}\left(\frac{m}{m_{0}}\right)\right\}^{\delta}\right]^{-b},
$$

where $\gamma=\left(0.5^{-1 / b}-1\right)^{-1 / \delta}$ and $m / m_{0}$ is the ratio of true median life to the specified median life.

The probability of rejecting a good lot is called the producer risk $\alpha$ and the probability of accepting a defective lot is called the consumer risk $\beta$. Group sampling plan under the $100 q^{\text {th }}$ percentile life to specified life $t_{q} / t_{q_{0}}$ is developed to find the minimum number of groups and acceptance number by satisfying the following two inequalities based on a two point approach such that consumer and producer risks are satisfied simultaneously.

$$
\begin{aligned}
& L\left(p \mid \frac{m}{m_{0}}=r_{1}\right) \leq \beta, \\
& L\left(p \mid \frac{m}{m_{0}}=r_{2}\right) \geq 1-\alpha,
\end{aligned}
$$


Table 1: Minimum number of groups and acceptance number for the Total failure plan for the Dagum distribution using percentile $q_{0.1}$ and $b=2, \delta=3$

\begin{tabular}{|c|c|c|c|c|c|c|c|c|c|c|c|c|c|}
\hline \multirow{3}{*}{$\beta$} & \multirow{3}{*}{$t_{q} / t_{q_{0}}$} & \multicolumn{6}{|c|}{$r=5$} & \multicolumn{6}{|c|}{$r=10$} \\
\hline & & \multicolumn{3}{|c|}{$\delta_{q}=0.5$} & \multicolumn{3}{|c|}{$\delta_{q}=1.0$} & \multicolumn{3}{|c|}{$\delta_{q}=0.5$} & \multicolumn{3}{|c|}{$\delta_{q}=1.0$} \\
\hline & & $g$ & $c$ & $\overline{\mathrm{L}(\mathrm{P} 2)}$ & $g$ & $c$ & $\mathrm{~L}(\mathrm{P} 2)$ & $g$ & $c$ & $\mathrm{~L}(\mathrm{P} 2)$ & $g$ & $c$ & $\mathrm{~L}(\mathrm{P} 2)$ \\
\hline \multirow{6}{*}{0.25} & 2 & 93 & 0 & 0.9764 & 3 & 0 & 0.9561 & 47 & 0 & 0.9761 & 3 & 1 & 0.9963 \\
\hline & 4 & 93 & 0 & 0.9996 & 3 & 0 & 0.9993 & 47 & 0 & 0.9996 & 2 & 0 & 0.9989 \\
\hline & 6 & 93 & 0 & 0.9999 & 3 & 0 & 0.9999 & 47 & 0 & 0.9999 & 2 & 0 & 0.9999 \\
\hline & 8 & 93 & 0 & 0.9999 & 3 & 0 & 0.9999 & 47 & 0 & 0.9999 & 2 & 0 & 0.9999 \\
\hline & 10 & 93 & 0 & 0.9999 & 3 & 0 & 0.9999 & 47 & 0 & 0.9999 & 2 & 0 & 0.9999 \\
\hline & 12 & 93 & 0 & 0.9999 & 3 & 0 & 0.9999 & 47 & 0 & 0.9999 & 2 & 0 & 0.9999 \\
\hline \multirow{6}{*}{0.10} & 2 & 154 & 0 & 0.9611 & 8 & 1 & 0.9935 & 77 & 0 & 0.9611 & 4 & 1 & 0.9935 \\
\hline & 4 & 154 & 0 & 0.9994 & 5 & 0 & 0.9987 & 77 & 0 & 0.9994 & 3 & 0 & 0.9985 \\
\hline & 6 & 154 & 0 & 0.9999 & 5 & 0 & 0.9999 & 77 & 0 & 0.9999 & 3 & 0 & 0.9998 \\
\hline & 8 & 154 & 0 & 0.9999 & 5 & 0 & 0.9999 & 77 & 0 & 0.9999 & 3 & 0 & 0.9999 \\
\hline & 10 & 154 & 0 & 0.9999 & 5 & 0 & 0.9999 & 77 & 0 & 0.9999 & 3 & 0 & 0.9999 \\
\hline & 12 & 154 & 0 & 0.9999 & 5 & 0 & 0.9999 & 77 & 0 & 0.9999 & 3 & 0 & 0.9999 \\
\hline \multirow{6}{*}{0.05} & 2 & 318 & 1 & 0.9968 & 10 & 1 & 0.9900 & 159 & 1 & 0.9968 & 5 & 1 & 0.9901 \\
\hline & 4 & 201 & 0 & 0.9992 & 6 & 0 & 0.9984 & 101 & 0 & 0.9992 & 3 & 0 & 0.9985 \\
\hline & 6 & 201 & 0 & 0.9999 & 6 & 0 & 0.9998 & 101 & 0 & 0.9999 & 3 & 0 & 0.9998 \\
\hline & 8 & 201 & 0 & 0.9999 & 6 & 0 & 0.9999 & 101 & 0 & 0.9999 & 3 & 0 & 0.9999 \\
\hline & 10 & 201 & 0 & 0.9999 & 6 & 0 & 0.9999 & 101 & 0 & 0.9999 & 3 & 0 & 0.9999 \\
\hline & 12 & 201 & 0 & 0.9999 & 6 & 0 & 0.9999 & 101 & 0 & 0.9999 & 3 & 0 & 0.9999 \\
\hline \multirow{6}{*}{0.01} & 2 & 444 & 1 & 0.9939 & 13 & 1 & 0.9836 & 222 & 1 & 0.9939 & 7 & 1 & 0.9812 \\
\hline & 4 & 308 & 0 & 0.9987 & 9 & 0 & 0.9977 & 154 & 0 & 0.9987 & 5 & 0 & 0.9974 \\
\hline & 6 & 308 & 0 & 0.9999 & 9 & 0 & 0.9998 & 154 & 0 & 0.9999 & 5 & 0 & 0.9998 \\
\hline & 8 & 308 & 0 & 0.9999 & 9 & 0 & 0.9999 & 154 & 0 & 0.9999 & 5 & 0 & 0.9999 \\
\hline & 10 & 308 & 0 & 0.9999 & 9 & 0 & 0.9999 & 154 & 0 & 0.9999 & 5 & 0 & 0.9999 \\
\hline & 12 & 308 & 0 & 0.9999 & 9 & 0 & 0.9999 & 154 & 0 & 0.9999 & 5 & 0 & 0.9999 \\
\hline
\end{tabular}

where $r_{1}$ and $r_{2}$ are the percentile ratios that will be specified by the consumer and producer risks, respectively. Let $p$ be the failure probabilities corresponding to the consumer and producer risks. The required number of groups can be determined through the following inequalities.

$$
\begin{aligned}
& L\left(p_{1}\right)=\left(\sum_{i=0}^{c}\left(\begin{array}{c}
r g \\
i
\end{array}\right) p_{1}^{i}\left(1-p_{1}\right)^{r g-i}\right) \leq \beta, \\
& L\left(p_{2}\right)=\left(\sum_{i=0}^{c}\left(\begin{array}{c}
r g \\
i
\end{array}\right) p_{2}^{i}\left(1-p_{2}\right)^{r g-i}\right) \geq 1-\alpha .
\end{aligned}
$$

\section{Description of Tables and Examples}

The constructed Table 1-Table 5 show the minimum number of groups $g$ and the acceptance number $c$ required for the proposed group acceptance sampling plan under the total failure according to various values of the consumer risk $(\beta=0.25,0.10,0.05,0.01)$ when the true percentile equals the specified life and $5 \%$ of producer's risk when the true percentiles $(2,4,6,8,10,12)$ times the specified life. Two level of group size $(r=5,10)$ and two levels of termination time multiplier $(a=0.5,1.0)$. We consider different values of the $b$ and $\delta$ to find the minimum sample size that can be obtained, if needed, by $n=r \times g$.

These tables are constructed by using different values of the shape parameter of Dagum distribution for example ( $b=2, \delta=3),(b=3, \delta=3),(b=0.40528, \delta=2.5214)$ to find the minimum number of groups and acceptance number by using the total failure plan. In Table 1 and Table 2 we use the $10^{\text {th }}$ percentile with shape parameters $(b=2, \delta=3),(b=3, \delta=3)$ to find the minimum number of groups and acceptance number for example $(g, c, \beta)=(93,0,0.25),(298,0,0.25)$ respectively. In Table 3 and Table 4 we use the $50^{\text {th }}$ percentile to find the minimum number of groups and acceptance number for example $(g, c, \beta)=(15,1,0.10),(14,0,0.10)$ respectively by using the shape parameters 
Table 2: Minimum number of groups and acceptance number for the Total failure plan for the Dagum distribution using percentile $q_{0.1}$ and $b=3, \delta=3$

\begin{tabular}{|c|c|c|c|c|c|c|c|c|c|c|c|c|c|}
\hline \multirow{3}{*}{$\beta$} & \multirow{3}{*}{$t_{q} / t_{q_{0}}$} & \multicolumn{6}{|c|}{$r=5$} & \multicolumn{6}{|c|}{$r=10$} \\
\hline & & \multicolumn{3}{|c|}{$\delta_{q}=0.5$} & \multicolumn{3}{|c|}{$\delta_{q}=1.0$} & \multicolumn{3}{|c|}{$\delta_{q}=0.5$} & \multicolumn{3}{|c|}{$\delta_{q}=1.0$} \\
\hline & & $g$ & $c$ & $\mathrm{~L}(\mathrm{P} 2)$ & $g$ & $\frac{T}{c}$ & $\mathrm{~L}(\mathrm{P} 2)$ & $g$ & c & $\mathrm{L}(\mathrm{P} 2)$ & $g$ & $c$ & $\mathrm{~L}(\mathrm{P} 2)$ \\
\hline \multirow{6}{*}{0.25} & 2 & 298 & 0 & 0.9964 & 3 & 0 & 0.9861 & $\begin{array}{c}0 \\
149\end{array}$ & 0 & 0.9964 & 2 & 0 & 0.9815 \\
\hline & 4 & 298 & 0 & 0.9999 & 3 & 0 & 0.9999 & 149 & 0 & 0.9999 & 2 & 0 & 0.9999 \\
\hline & 6 & 298 & 0 & 0.9999 & 3 & 0 & 0.9999 & 149 & 0 & 0.9999 & 2 & 0 & 0.9999 \\
\hline & 8 & 298 & 0 & 0.9999 & 3 & 0 & 0.9999 & 149 & 0 & 0.9999 & 2 & 0 & 0.9999 \\
\hline & 10 & 298 & 0 & 0.9999 & 3 & 0 & 0.9999 & 149 & 0 & 0.9999 & 2 & 0 & 0.9999 \\
\hline & 12 & 298 & 0 & 1.0000 & 3 & 0 & 0.9999 & 149 & 0 & 0.9999 & 2 & 0 & 0.9999 \\
\hline \multirow{6}{*}{0.10} & 2 & 494 & 0 & 0.9941 & 5 & 0 & 0.9769 & 247 & 0 & 0.9941 & 3 & 0 & 0.9724 \\
\hline & 4 & 494 & 0 & 0.9999 & 5 & 0 & 0.9999 & 247 & 0 & 0.9999 & 3 & 0 & 0.9999 \\
\hline & 6 & 494 & 0 & 0.9999 & 5 & 0 & 0.9999 & 247 & 0 & 0.9999 & 3 & 0 & 0.9999 \\
\hline & 8 & 494 & 0 & 0.9999 & 5 & 0 & 0.9999 & 247 & 0 & 0.9999 & 3 & 0 & 0.9999 \\
\hline & 10 & 494 & 0 & 0.9999 & 5 & 0 & 0.9999 & 247 & 0 & 0.9999 & 3 & 0 & 0.9999 \\
\hline & 12 & 494 & 0 & 0.9999 & 5 & 0 & 0.9999 & 247 & 0 & 0.9999 & 3 & 0 & 0.9999 \\
\hline \multirow{6}{*}{0.05} & 2 & 642 & 0 & 0.9924 & 6 & 0 & 0.9724 & 321 & 0 & 0.9924 & 3 & 0 & 0.9724 \\
\hline & 4 & 642 & 0 & 0.9999 & 6 & 0 & 0.9999 & 321 & 0 & 0.9999 & 3 & 0 & 0.9999 \\
\hline & 6 & 642 & 0 & 0.9999 & 6 & 0 & 0.9999 & 321 & 0 & 0.9999 & 3 & 0 & 0.9999 \\
\hline & 8 & 642 & 0 & 0.9999 & 6 & 0 & 0.9999 & 321 & 0 & 0.9999 & 3 & 0 & 0.9999 \\
\hline & 10 & 642 & 0 & 0.9999 & 6 & 0 & 0.9999 & 321 & 0 & 0.9999 & 3 & 0 & 0.9999 \\
\hline & 12 & 642 & 0 & 0.9999 & 6 & 0 & 0.9999 & 321 & 0 & 0.9999 & 3 & 0 & 0.9999 \\
\hline \multirow{6}{*}{0.01} & 2 & 987 & 0 & 0.9883 & 9 & 0 & 0.9588 & 494 & 0 & 0.9883 & 5 & 0 & 0.9544 \\
\hline & 4 & 987 & 0 & 0.9999 & 9 & 0 & 0.9998 & 494 & 0 & 0.9999 & 5 & 0 & 0.9998 \\
\hline & 6 & 987 & 0 & 0.9999 & 9 & 0 & 0.9999 & 494 & 0 & 0.9999 & 5 & 0 & 0.9999 \\
\hline & 8 & 987 & 0 & 0.9999 & 9 & 0 & 0.9999 & 494 & 0 & 0.9999 & 5 & 0 & 0.9999 \\
\hline & 10 & 987 & 0 & 0.9999 & 9 & 0 & 0.9999 & 494 & 0 & 0.9999 & 5 & 0 & 0.9999 \\
\hline & 12 & 987 & 0 & 0.9999 & 9 & 0 & 0.9999 & 494 & 0 & 0.9999 & 5 & 0 & 0.99996 \\
\hline
\end{tabular}

Table 3: Minimum number Of groups and acceptance number for the Total failure plan for the Dagum distribution using percentile $q_{0.5}$ and $b=2, \delta=3$

\begin{tabular}{|c|c|c|c|c|c|c|c|c|c|c|c|c|c|}
\hline \multirow{3}{*}{$\beta$} & \multirow{3}{*}{$m / m_{0}$} & \multicolumn{6}{|c|}{$r=5$} & \multicolumn{6}{|c|}{$r=10$} \\
\hline & & \multicolumn{3}{|c|}{$\delta_{q}=0.5$} & \multicolumn{3}{|c|}{$\delta_{q}=1.0$} & \multicolumn{3}{|c|}{$\delta_{q}=0.5$} & \multicolumn{3}{|c|}{$\delta_{q}=1.0$} \\
\hline & & $g$ & $c$ & $\mathrm{~L}(\mathrm{P} 2)$ & $g$ & $c$ & $\mathrm{~L}(\mathrm{P} 2)$ & $g$ & $c$ & $\mathrm{~L}(\mathrm{P} 2)$ & $g$ & $c$ & $\mathrm{~L}(\mathrm{P} 2)$ \\
\hline \multirow{6}{*}{0.25} & 2 & 6 & 0 & 0.9612 & 1 & 1 & 0.9741 & 3 & 0 & 0.9612 & 1 & 2 & 0.9860 \\
\hline & 4 & 6 & 0 & 0.9993 & 1 & 0 & 0.9934 & 3 & 0 & 0.9993 & 1 & 0 & 0.9868 \\
\hline & 6 & 6 & 0 & 0.9999 & 1 & 0 & 0.9994 & 3 & 0 & 0.9999 & 1 & 0 & 0.9988 \\
\hline & 8 & 6 & 0 & 0.9999 & 1 & 0 & 0.9999 & 3 & 0 & 0.9999 & 1 & 0 & 0.9998 \\
\hline & 10 & 6 & 0 & 0.9999 & 1 & 0 & 0.9999 & 3 & 0 & 0.9999 & 1 & 0 & 0.9999 \\
\hline & 12 & 6 & 0 & 0.9999 & 1 & 0 & 0.9999 & 3 & 0 & 0.9999 & 1 & 0 & 0.9999 \\
\hline \multirow{6}{*}{0.10} & 2 & 15 & 1 & 0.9955 & 2 & 2 & 0.9860 & 8 & 1 & 0.9948 & 1 & 2 & 0.9860 \\
\hline & 4 & 9 & 0 & 0.9990 & 1 & 0 & 0.9934 & 5 & 0 & 0.9989 & 1 & 0 & 0.9868 \\
\hline & 6 & 9 & 0 & 0.9999 & 1 & 0 & 0.9994 & 5 & 0 & 0.9999 & 1 & 0 & 0.9988 \\
\hline & 8 & 9 & 0 & 0.9999 & 1 & 0 & 0.9999 & 5 & 0 & 0.9999 & 1 & 0 & 0.9998 \\
\hline & 10 & 9 & 0 & 0.9999 & 1 & 0 & 0.9999 & 5 & 0 & 0.9999 & 1 & 0 & 0.9999 \\
\hline & 12 & 9 & 0 & 0.9999 & 1 & 0 & 0.9999 & 5 & 0 & 0.9999 & 1 & 0 & 0.9999 \\
\hline \multirow{6}{*}{0.05} & 2 & 18 & 1 & 0.9935 & 3 & 2 & 0.9566 & 9 & 1 & 0.9935 & 2 & 3 & 0.9797 \\
\hline & 4 & 11 & 0 & 0.9988 & 1 & 0 & 0.9934 & 6 & 0 & 0.9987 & 1 & 0 & 0.9868 \\
\hline & 6 & 11 & 0 & 0.9999 & 1 & 0 & 0.9994 & 6 & 0 & 0.9999 & 1 & 0 & 0.9988 \\
\hline & 8 & 11 & 0 & 0.9999 & 1 & 0 & 0.9999 & 6 & 0 & 0.9999 & 1 & 0 & 0.9998 \\
\hline & 10 & 11 & 0 & 0.9999 & 1 & 0 & 0.9999 & 6 & 0 & 0.9999 & 1 & 0 & 0.9999 \\
\hline & 12 & 11 & 0 & 0.9999 & 1 & 0 & 0.9999 & 6 & 0 & 0.9999 & 1 & 0 & 0.9999 \\
\hline \multirow{6}{*}{0.01} & 2 & 25 & 1 & 0.9878 & 3 & 2 & 0.9566 & 13 & 1 & 0.9869 & 2 & 3 & 0.9797 \\
\hline & 4 & 17 & 0 & 0.9981 & 2 & 0 & 0.9868 & 9 & 0 & 0.9980 & 1 & 0 & 0.9868 \\
\hline & 6 & 17 & 0 & 0.9998 & 2 & 0 & 0.9988 & 9 & 0 & 0.9998 & 1 & 0 & 0.9988 \\
\hline & 8 & 17 & 0 & 0.9999 & 2 & 0 & 0.9998 & 9 & 0 & 0.9999 & 1 & 0 & 0.9998 \\
\hline & 10 & 17 & 0 & 0.9999 & 2 & 0 & 0.9999 & 9 & 0 & 0.9999 & 1 & 0 & 0.9999 \\
\hline & 12 & 17 & 0 & 0.9999 & 2 & 0 & 0.9999 & 9 & 0 & 0.9999 & 1 & 0 & 0.9999 \\
\hline
\end{tabular}

$(b=2, \delta=3),(b=3, \delta=3)$.

Suppose that the lifetime of a product under consideration is known to follow a Dagum distribution with the shape parameter $(b=2, \delta=3)$. Suppose that it is desired to develop a group sampling plan 
Table 4: Minimum number Of groups and acceptance number for the Total failure plan for the Dagum distribution using percentile $q_{0.5}$ and $b=3, \delta=3$

\begin{tabular}{|c|c|c|c|c|c|c|c|c|c|c|c|c|c|}
\hline \multirow{3}{*}{$\beta$} & \multirow{3}{*}{$m / m_{0}$} & \multicolumn{6}{|c|}{$r=5$} & \multicolumn{6}{|c|}{$r=10$} \\
\hline & & \multicolumn{3}{|c|}{$\delta_{q}=0.5$} & \multicolumn{3}{|c|}{$\delta_{q}=1.0$} & \multicolumn{3}{|c|}{$\delta_{q}=0.5$} & \multicolumn{3}{|c|}{$\delta_{q}=1.0$} \\
\hline & & $g$ & $c$ & $\mathrm{~L}(\mathrm{P} 2)$ & $g$ & $\frac{4}{c}$ & $\mathrm{~L}(\mathrm{P} 2)$ & $g$ & $c$ & $\mathrm{~L}(\mathrm{P} 2)$ & $g$ & $\frac{4}{c}$ & $\mathrm{~L}(\mathrm{P} 2)$ \\
\hline \multirow{6}{*}{0.25} & 2 & 8 & 0 & 0.9927 & 1 & 1 & 0.9890 & 4 & 0 & 0.9927 & 1 & 1 & 0.9560 \\
\hline & 4 & 8 & 0 & 0.9999 & 1 & 0 & 0.9991 & 4 & 0 & 0.9999 & 1 & 0 & 0.9982 \\
\hline & 6 & 8 & 0 & 0.9999 & 1 & 0 & 0.9999 & 4 & 0 & 0.9999 & 1 & 0 & 0.9999 \\
\hline & 8 & 8 & 0 & 0.9999 & 1 & 0 & 0.9999 & 4 & 0 & 0.9999 & 1 & 0 & 0.9999 \\
\hline & 10 & 8 & 0 & 0.9999 & 1 & 0 & 0.9999 & 4 & 0 & 0.9999 & 1 & 0 & 0.9999 \\
\hline & 12 & 8 & 0 & 0.9999 & 1 & 0 & 0.9999 & 4 & 0 & 0.9999 & 1 & 0 & 0.9999 \\
\hline \multirow{6}{*}{0.10} & 2 & 14 & 0 & 0.9874 & 2 & 1 & 0.9560 & 7 & 0 & 0.9874 & 1 & 1 & 0.9560 \\
\hline & 4 & 14 & 0 & 0.9999 & 1 & 0 & 0.9991 & 7 & 0 & 0.9999 & 1 & 0 & 0.9982 \\
\hline & 6 & 14 & 0 & 0.9999 & 1 & 0 & 0.9999 & 7 & 0 & 0.9999 & 1 & 0 & 0.9999 \\
\hline & 8 & 14 & 0 & 0.9999 & 1 & 0 & 0.9999 & 7 & 0 & 0.9999 & 1 & 0 & 0.9999 \\
\hline & 10 & 14 & 0 & 0.9999 & 1 & 0 & 0.9999 & 7 & 0 & 0.9999 & 1 & 0 & 0.9999 \\
\hline & 12 & 14 & 0 & 0.9999 & 1 & 0 & 0.9999 & 7 & 0 & 0.9999 & 1 & 0 & 0.9999 \\
\hline \multirow{6}{*}{0.05} & 2 & 18 & 0 & 0.9837 & 2 & 1 & 0.9560 & 9 & 0 & 0.9837 & 1 & 1 & 0.9560 \\
\hline & 4 & 18 & 0 & 0.9999 & 1 & 0 & 0.9991 & 9 & 0 & 0.9999 & 1 & 0 & 0.9982 \\
\hline & 6 & 18 & 0 & 0.9999 & 1 & 0 & 0.9999 & 9 & 0 & 0.9999 & 1 & 0 & 0.9999 \\
\hline & 8 & 18 & 0 & 0.9999 & 1 & 0 & 0.9999 & 9 & 0 & 0.9999 & 1 & 0 & 0.9999 \\
\hline & 10 & 18 & 0 & 0.9999 & 1 & 0 & 0.9999 & 9 & 0 & 0.9999 & 1 & 0 & 0.9999 \\
\hline & 12 & 18 & 0 & 0.9999 & 1 & 0 & 0.9999 & 9 & 0 & 0.9999 & 1 & 0 & 0.9999 \\
\hline \multirow{6}{*}{0.01} & 2 & 27 & 0 & 0.9757 & 3 & 2 & 0.9866 & 14 & 0 & 0.9748 & 2 & 2 & 0.9704 \\
\hline & 4 & 27 & 0 & 0.9999 & 2 & 0 & 0.9982 & 14 & 0 & 0.9999 & 1 & 0 & 0.9982 \\
\hline & 6 & 27 & 0 & 0.9999 & 2 & 0 & 0.9999 & 14 & 0 & 0.9999 & 1 & 0 & 0.9999 \\
\hline & 8 & 27 & 0 & 0.9999 & 2 & 0 & 0.9999 & 14 & 0 & 0.9999 & 1 & 0 & 0.9999 \\
\hline & 10 & 27 & 0 & 0.9999 & 2 & 0 & 0.9999 & 14 & 0 & 0.9999 & 1 & 0 & 0.9999 \\
\hline & 12 & 27 & 0 & 0.9999 & 2 & 0 & 0.9999 & 14 & 0 & 0.9999 & 1 & 0 & 0.9999 \\
\hline
\end{tabular}

Table 5: Minimum number of groups and acceptance number for the Total failure plan for the Dagum distribution using percentile $q_{0.1}$ and $b=0.40528, \delta=2.5214$

\begin{tabular}{|c|c|c|c|c|c|c|c|c|c|c|c|c|c|}
\hline \multirow{3}{*}{$\beta$} & \multirow{3}{*}{$t_{q} / t_{q_{0}}$} & \multicolumn{6}{|c|}{$r=5$} & \multicolumn{6}{|c|}{$r=10$} \\
\hline & & \multicolumn{3}{|c|}{$\delta_{q}=0.5$} & \multicolumn{3}{|c|}{$\delta_{q}=1.0$} & \multicolumn{3}{|c|}{$\delta_{q}=0.5$} & \multicolumn{3}{|c|}{$\delta_{q}=1.0$} \\
\hline & & $g$ & $c$ & $\mathrm{~L}(\mathrm{P} 2)$ & $g$ & $c$ & $\mathrm{~L}(\mathrm{P} 2)$ & $g$ & $c$ & $\mathrm{~L}(\mathrm{P} 2)$ & $g$ & $c$ & $\mathrm{~L}(\mathrm{P} 2)$ \\
\hline \multirow{6}{*}{0.25} & 2 & - & - & - & - & - & - & - & - & - & - & - & - \\
\hline & 4 & 21 & 3 & 0.9622 & 11 & 3 & 0.9554 & 11 & 3 & 0.9564 & 7 & 4 & 0.9722 \\
\hline & 6 & 16 & 2 & 0.9742 & 8 & 2 & 0.9737 & 8 & 2 & 0.9742 & 4 & 2 & 0.9737 \\
\hline & 8 & 11 & 1 & 0.9581 & 6 & 1 & 0.9502 & 6 & 1 & 0.9509 & 3 & 1 & 0.9502 \\
\hline & 10 & 11 & 1 & 0.9723 & 6 & 1 & 0.9669 & 6 & 1 & 0.9675 & 3 & 1 & 0.9669 \\
\hline & 12 & 11 & 1 & 0.9804 & 6 & 1 & 0.9765 & 6 & 1 & 0.9769 & 3 & 1 & 0.9765 \\
\hline \multirow{6}{*}{0.10} & 2 & - & - & - & - & - & - & - & - & - & - & - & - \\
\hline & 4 & 33 & 4 & 0.9508 & 16 & 4 & 0.9545 & 19 & 5 & 0.9723 & 8 & 4 & 0.9545 \\
\hline & 6 & 27 & 3 & 0.9772 & 13 & 3 & 0.9793 & 14 & 3 & 0.9743 & 7 & 3 & 0.9736 \\
\hline & 8 & 22 & 2 & 0.9723 & 11 & 2 & 0.9717 & 11 & 2 & 0.9723 & 6 & 2 & 0.9646 \\
\hline & 10 & 22 & 2 & 0.9847 & 11 & 2 & 0.9843 & 11 & 2 & 0.9847 & 6 & 2 & 0.9803 \\
\hline & 12 & 16 & 1 & 0.9608 & 8 & 1 & 0.9600 & 8 & 1 & 0.9608 & 4 & 1 & 0.9600 \\
\hline \multirow{6}{*}{0.05} & 2 & - & - & - & - & - & - & - & - & - & - & - & - \\
\hline & 4 & 43 & 5 & 0.9541 & 21 & 5 & 0.9566 & 24 & 6 & 0.9734 & 12 & 6 & 0.9724 \\
\hline & 6 & 31 & 3 & 0.9646 & 16 & 3 & 0.9598 & 16 & 3 & 0.9610 & 8 & 3 & 0.9598 \\
\hline & 8 & 26 & 2 & 0.9578 & 13 & 2 & 0.9568 & 13 & 2 & 0.9578 & 8 & 3 & 0.9843 \\
\hline & 10 & 26 & 2 & 0.9763 & 13 & 2 & 0.9756 & 13 & 2 & 0.9763 & 7 & 2 & 0.9705 \\
\hline & 12 & 26 & 2 & 0.9854 & 13 & 2 & 0.9850 & 13 & 2 & 0.9854 & 7 & 2 & 0.9817 \\
\hline \multirow{6}{*}{0.01} & 2 & - & - & - & - & - & - & - & - & - & - & - & - \\
\hline & 4 & - & - & - & - & - & - & 32 & 7 & 0.9595 & 16 & 7 & 0.9574 \\
\hline & 6 & 47 & 4 & 0.9599 & 23 & 4 & 0.9615 & 24 & 4 & 0.9568 & 12 & 4 & 0.9552 \\
\hline & 8 & 41 & 3 & 0.9660 & 20 & 3 & 0.9675 & 21 & 3 & 0.9634 & 10 & 3 & 0.9675 \\
\hline & 10 & 34 & 2 & 0.9534 & 17 & 2 & 0.9521 & 17 & 2 & 0.9534 & 10 & 3 & 0.9843 \\
\hline & 12 & 34 & 2 & 0.9706 & 17 & 2 & 0.9698 & 17 & 2 & 0.9706 & 9 & 2 & 0.9651 \\
\hline
\end{tabular}

Note: (-) shows plan parameters does not exist.

based on the total number of failures to assure that the percentile life is greater than 500h through the experiment to be completed by 500h using testers equipped with five products each. It is assumed that the consumer risk is $10 \%$ when the true percentile is $500 \mathrm{~h}$ and the producer risk is $5 \%$ when the true 
Table 6: Comparison between proposed plan vs. existing plan when $b=2, \delta=3, \delta_{q}=1.0$

\begin{tabular}{|c|c|c|c|c|c|}
\hline \multirow{3}{*}{$\beta$} & \multirow{3}{*}{$t_{q} / t_{q_{0}}$} & \multicolumn{2}{|c|}{$\begin{array}{c}q_{0.1} \\
\text { Dagum distribution }\end{array}$} & \multicolumn{2}{|c|}{$\frac{q_{0.5}}{\text { Dagum distribution }}$} \\
\hline & & Total failure plan & existing plan & Total failure plan & existing plan \\
\hline & & $g$ & $g$ & $g$ & $g$ \\
\hline \multirow{3}{*}{0.25} & 2 & 3 & 3 & 1 & 1 \\
\hline & 4 & 3 & 3 & 1 & 1 \\
\hline & 6 & 3 & 3 & 1 & 1 \\
\hline \multirow{3}{*}{0.10} & 2 & 8 & 28 & 2 & 4 \\
\hline & 4 & 5 & 5 & 1 & 1 \\
\hline & 6 & 5 & 5 & 1 & 1 \\
\hline \multirow{3}{*}{0.05} & 2 & 10 & 36 & 3 & 5 \\
\hline & 4 & 6 & 6 & 1 & 1 \\
\hline & 6 & 6 & 6 & 1 & 1 \\
\hline \multirow{3}{*}{0.01} & 2 & 13 & 55 & 3 & 7 \\
\hline & 4 & 9 & 9 & 2 & 2 \\
\hline & 6 & 9 & 9 & 2 & 2 \\
\hline
\end{tabular}

Table 7: Comparison between Dagum distribution vs. Marshall-Olkin Extended Lomax distribution when $b=3, \delta=3$ and $b=5, \delta_{q}=1.0$

\begin{tabular}{|c|c|c|c|c|c|}
\hline \multirow{3}{*}{$\beta$} & \multirow{3}{*}{$t_{q} / t_{q_{0}}$} & \multicolumn{2}{|c|}{$q_{0.1}$} & \multicolumn{2}{|c|}{$q_{0.5}$} \\
\hline & & Dagum distribution & Marshall-Olkin & Dagum distribution & Marshall-Olkin \\
\hline & & $g$ & $g$ & $g$ & $g$ \\
\hline \multirow{3}{*}{0.25} & 2 & 3 & - & 1 & - \\
\hline & 4 & 3 & 11 & 1 & 2 \\
\hline & 6 & 3 & 8 & 1 & 2 \\
\hline \multirow{3}{*}{0.10} & 2 & 5 & - & 2 & - \\
\hline & 4 & 5 & 19 & 1 & - \\
\hline & 6 & 5 & 13 & 1 & 3 \\
\hline \multirow{3}{*}{0.05} & 2 & 6 & - & 2 & - \\
\hline & 4 & 6 & 21 & 1 & - \\
\hline & 6 & 6 & 16 & 1 & 4 \\
\hline \multirow{3}{*}{0.01} & 2 & 9 & - & 3 & - \\
\hline & 4 & 9 & - & 2 & - \\
\hline & 6 & 9 & 23 & 2 & 4 \\
\hline
\end{tabular}

percentile is $1000 \mathrm{~h}$. Since $(b=2, \delta=3) \beta=0.10, r=5, a=1.0, t_{q} / t_{q_{0}}=4$ the minimum number of groups and acceptance number can be found as $g=5$ and $c=0$ Table 1 . This means that a total of 25 products are needed and that five items will be allocated to each of the five testers. We will accept the lots if no more than zero failures occur before 500h in all groups combine, otherwise the lot is rejected.

\section{Comparison}

In The Table 6, the number of groups finding by a total failure plan is minimal when compared to an existing plan. The number of groups of Level 2 is minimum in the total failure plan when compared to the existing plan. In Table 7, Dagum distribution is compared to Marshall-Olkin Extended Lomax distribution and it is observed that the number of groups from the Dagum distribution is minimal when as compared to the Marshall-Olkin Extended Lomax distribution. 


\section{Example}

Let an experimenter want to establish the true unknown $10^{\text {th }}$ percentile for the small electric (Lio et al., 2010b) cart mentioned below to be at least 2 months and the life test would end at 6 months, this should have led to the ratio $t_{q} / t_{q_{0}}=8$. Lifetime (in months) to first failure of 20 electric carts is:

$$
0.9,1.5,2.3,3.2,3.9,5.0,6.2,7.5,8.3,10.4,11.1,12.6,15,16.3,19.3,22.6,24.8,31.5,38.1,53.0
$$

The Kolmogorov-Smirnov test is fitted by these twenty observations as the $p$-value is 0.190 , this $p$-value indicated that the Dagum distribution as a reasonable goodness of fit for these twenty observations. The maximum likelihood estimate(MLE) of the unknown shape parameter of the Dagum distribution for these twenty observations is $b=0.40528, \delta=2.5214$, respectively. Thus, with $c=3$ and $\beta=0.01$, the experimenter should find from Table 5 that the number of group must be at least 20 and the sampling plan $\left(g, c, a, t_{q} / t_{q_{0}}\right)=(20,3,1.0 .8)$. Since there were six items with a failure time of less than or equal to 6 months in the given group size of 20 observations, the experiment would reject the null hypothesis and accept that the $10^{\text {th }}$ percentile lifetime of less than 2 with a confidence $\beta=0.01$.

\section{Conclusion}

This paper develop a group acceptance sampling plan for Dagum distribution under the percentile lifetime by using the total failure plan. The required minimum number of groups and acceptance numbers are determined by using a two point approach. This paper only deals with the Dagum distribution to check the multiple numbers of items simultaneously by saving cost and time. The number of group obtained from the proposed plan are less than the existing group plan that indicates that the proposed plan is superior than the existing plan (stated by Aslam and Jun, 2009b) in saving cost and time truncated associated with the life tests. Further, results of the proposed plan shows that the Dagum distribution is better than the Marshall-Olkin Extended Lomax distribution at $10^{\text {th }}$ and $50^{\text {th }}$ percentile. The present paper can be extended for other distributions through future research.

\section{Acknowledgements}

The authors are deeply thankful to the editor and reviewers for their valuable comments to improve the manuscript.

\section{References}

Aslam, M., (2008). Economic reliability acceptance sampling plan for generalized Rayleigh distribution, Journal of Statistics, 15, 26-35.

Aslam, M. and Jun, C.-H. (2009a). A group acceptance sampling plan for truncated life test having Weibull distribution, Journal of Applied Statistics (UK), 39, 1021-1027.

Aslam, M. and Jun, C. H. (2009b). A group acceptance sampling plans for truncated life tests based on the inverse Rayleigh and log-logistic distributions, Pakistan Journal of Statistics, 25, 107-119.

Aslam, M. and Jun, C. H. (2010). A double acceptance sampling plan for generalized log-logistic distributions with known shape parameters, Journal of Applied Statistics, 37, 405-414.

Aslam, M. and Kantam, R. R. L. (2008). Economic reliability acceptance sampling based on truncated life tests in the Birnbaum-Saunders distribution, Pakistan Journal of Statistics, 24, 269-276.

Aslam, M. and Shahbaz, M. Q. (2007).Economic Reliability Tests Plans using the Generalized Exponential Distribution, Journal of Statistics, 14, 52-59. 
Baklizi, A. (2003). Acceptance sampling based on truncated life tests in the Pareto distribution of the second kind, Advances and Applications in Statistics, 3, 33-48.

Balakrishnan, N., Leiva, V. and Lopez, J. (2007). Acceptance sampling plans from truncated life tests based on the generalized Birnbaum-Saunders distribution, Communications in Statistics Simulation and Computation, 36, 643-656.

Dagum, C. (1977). A new model of personal income distribution: specification and estimation, Economic Appliquee, 30, 413-437.

Domma, F. G., Latorre and Zenga, M. (2009). Reliability studies of Dagum distribution, submitted.

Epstein, B. (1954). Truncated life tests in the exponential case, Annals of Mathematical Statistics, 25, $555-564$.

Fertig, F. W. and Mann, N. R. (1980). Life test sampling plans for two parameter Weibull populations, Technometrics, 22, 165-177.

Goode, H. P. and Kao, J. H. K. (1961). Sampling plans based on the Weibull distribution, In Proceeding of the Seventh National Symposium on Reliability and Quality Control, (24-40). Philadelphia.

Jun, C. H., Balamurali, S. and Lee, S. H. (2006). Variables sampling plans for Weibull distribution lifetimes under sudden death testing, IEEE Transactions on Reliability, 55, 53-58.

Kantam, R. R. L. and Rosaiah, K. (1998). Half logistic distribution in acceptance sampling based on life tests, IAPQR Transactions, 23, 117-125.

Kantam, R. R. L., Rosaiah, K. and Rao, G. S. (2001). Acceptance sampling based on life tests: Log-logistic models, Journal of Applied Statistics, 28, 121-128.

Lio, Y. L., Tsai, Tzong-Ru and Wu, Shuo-Jye. (2010a). Acceptance sampling plans from truncated life tests based on the Birnbaum-saunders distribution for Percentiles, Communications in Statistics - Simulation and Computation, 39, 119-136.

Lio, Y. L., Tsai, Tzong-Ru and Wu, Shuo-Jye. (2010b). Acceptance sampling plans from truncated life tests based on Burr type XII percentiles, Journal of Chinese institute of Industrial Engineers, 27, 270-280.

Pascual, F. G. and Meeker, W. Q. (1998). The modified sudden death test: Planning life tests with a limited number of test positions, Journal of Testing and Evaluation, 26, 434-443.

Rosaiah, K. and Kantam, R. R. L. (2005). Acceptance sampling based on the inverse Rayleigh distribution, Economic Quality Control, 20, 277-286.

Rosaiah, K., Kantam, R. R. L. and Santosh Kumar, Ch. (2006). Reliability of test plans for exponentiated log-logistic distribution, Eco.Quality Control, 21, 165-175.

Rosaiah, K., Kantam, R. R. L. and Santosh Kumar, Ch. (2007). Exponentiated log-logistic distributionAn economic reliability test plan, Pakistan Journal of Statistics, 23, 147-146.

Srinivasa Rao, G., Ghitany, M. E., Kantam, R. R. L. (2009). Acceptance sampling plans for MarshalOlkin extended Lomax distribution, International Journal of Applied Mathematics, 22, 139-148.

Tsai, T. R. and Wu, S. J. (2006). Acceptance sampling based on truncated life tests for generalized Rayleigh distribution, Journal of Applied Statistics, 33, 595-600.

Vleek, B. L., Hendricks, R. C. and Zaretsky, E. V. (2003). Monto Carlo simulation of Sudden Death Bearing Testing, NASA, Hanover, MD, USA. 